\title{
A stable/unstable manifold theorem for local homeomorphisms of the plane
}

\author{
FRANCISCO R. RUIZ DEL PORTAL $\dagger$ and JOSÉ M. SALAZAR $\ddagger$ \\ $\dagger$ Departamento de Geometría y Topología, Facultad de CC. Matemáticas, \\ Universidad Complutense de Madrid, Madrid 28040, Spain \\ (e-mail: r_portal@mat.ucm.es) \\ † Departamento de Matemáticas, Universidad de Alcalá, \\ Alcalá de Henares, Madrid 28871, Spain \\ (e-mail: josem.salazar@uah.es)
}

(Received 29 April 2003 and accepted in revised form 11 February 2004)

\begin{abstract}
We use a notion (introduced in Topology 41 (2002), 1119-1212), which is stronger than the concept of filtration pair, to prove a stable/unstable manifold general theorem for local homeomorphisms of the plane in a neighborhood of an isolated fixed point.
\end{abstract}

\section{Introduction and preliminary definitions}

The stable and unstable manifold theorem for hyperbolic diffeomorphisms plays a very important role in differential dynamics. At the topological level Baldwin and Slaminka proved, in [1], a stable/unstable manifold theorem for area- and orientationpreserving homeomorphisms of orientable 2-manifolds having isolated fixed points of index less than 1 .

There are many papers in the literature relating the fixed-point index of a homeomorphism $f$ in a neighborhood of an isolated fixed point, and the local dynamical behavior of $f$. There are results in both directions, i.e. bounds (or explicit computation) for the fixed-point index from dynamical properties of $f$ and how the knowledge of the fixed-point index can be applied to describe the dynamics locally. We will mention besides [1], for example, the papers of Bonino [2], Brown [6], Le Calvez [9], Le Calvez and Yoccoz [10], Dancer and Ortega [12], Hirsch [17], Pelikan and Slaminka [20], Simon [23] and Shub and Sullivan [22], for their relations with the present paper. Their results frequently deal with orientation-preserving homeomorphisms. The main reason for this assumption is the application of some version of Brouwer's lemma on translation arcs, see [5] or [7].

The aim of this paper is to use the fixed-point index to obtain information about the dynamical behavior of a planar local homeomorphism in a neighborhood of an 
isolated fixed point. We will work with both the orientation-preserving and -reversing cases. The main tool we will use is a notion stronger than that of filtration pair which we introduced in [21]. This notion, based on the concept of filtration, see [15], allows us to apply Conley index ideas to the computation of the fixed-point index in the plane. Le Calvez and Yoccoz proved, in [10], the non-existence of minimal homeomorphisms of $\mathbb{R}^{2} \backslash K$, for any finite set $K$, using fixed-point index theory (see also [14] and [16]). Given an orientation-preserving local homeomorphism $f: U \subset \mathbb{R}^{2} \rightarrow \mathbb{R}^{2}$ they made a strong local study near a fixed point $p$ which is a locally maximal invariant set and which is neither a sink nor a source, which allowed them to prove the existence of integers $r, q \geq 1$ such that

$$
i_{\mathbb{R}^{2}}\left(f^{k}, p\right)= \begin{cases}1-r q & \text { if } k \in r \mathbb{N}, \\ 1 & \text { if } k \notin r \mathbb{N} .\end{cases}
$$

The present authors, in [21], by means of filtration pairs, gave in a simpler and more elementary way a general theorem that extends the above result to arbitrary local homeomorphisms. In particular, if $f$ reverses the orientation, there are integers $\delta \in$ $\{0,1,2\}$ and $q$ such that

$$
i_{\mathbb{R}^{2}}\left(f^{k}, p\right)= \begin{cases}1-\delta & \text { if } k \text { odd } \\ 1-\delta-2 q & \text { if } k \text { even. }\end{cases}
$$

Recently Bonino (see [2]) proved that $i_{\mathbb{R}^{2}}(f, p) \in\{0,1,-1\}$ for orientation-reversing planar homeomorphisms $f$ such that $p$ is an isolated fixed point.

As we will show, the existence of nice filtration pairs provides important information about the local dynamics in a neighborhood of an isolated fixed point. This kind of index pair is a tool that can be applied to orientation-preserving and orientation-reversing homeomorphisms and they exist under a more general setting than that of the above result (see Theorem 3). In the Main Theorem of this paper and its corollaries we will give some topological properties of the stable and unstable manifolds in a neighborhood of an isolated fixed point that is contained in a compact invariant set that admits such a nice filtration pair. Taking into account that $\left|i_{\mathbb{R}^{2}}\left(f^{-1}, p\right)\right|=\left|i_{\mathbb{R}^{2}}(f, p)\right|$, all of our results can be stated for $f^{-1}$. Then the properties of the stable manifold are naturally extended for the unstable one.

Let us state some of the definitions that we will need consistently. Let $U \subset X$ be an open set. By a (local) semidynamical system we mean a locally defined continuous map $f: U \rightarrow X$. The invariant part of $N, \operatorname{Inv}(N, f)$, is defined as the set of all $x \in N$ such that there is a full orbit $\gamma$ with $x \in \gamma \subset N$.

$\operatorname{Inv}^{+}(M, f)$ (respectively $\operatorname{Inv}^{-}(M, f)$ ) will denote the set of all $x \in M$ such that $f^{j}(x) \in M$ for every $j \in \mathbb{N}$ (respectively $f^{-j}(x)$ is well defined and belongs to $M$ for every $j \in \mathbb{N}$ ). Given $x \in U$, we will write

$$
\Lambda^{+}(x)=\left\{y \in U:\left\{f^{k}(y)\right\}_{k \in \mathbb{N}} \text { is well defined and } \lim _{k \rightarrow \infty} f^{k}(y)=x\right\}
$$

and

$$
\Lambda^{-}(x)=\left\{y \in U:\left\{f^{-k}(y)\right\}_{k \in \mathbb{N}} \text { is well defined and } \lim _{k \rightarrow \infty} f^{-k}(y)=x\right\}
$$


A compact set $S \subset X$ is invariant if $f(S)=S$. A compact invariant set $S$ is isolated with respect to $f$ if there exists a compact neighborhood $N$ of $S \operatorname{such}$ that $\operatorname{Inv}(N, f)=S$. The neighborhood $N$ is called an isolating neighborhood of $S$. If $X$ is a locally compact ANR (absolute neighborhood retract for metric spaces), $i_{X}(f, S)$ will denote the fixedpoint index of $f$ in a small enough neighborhood of $S$. The reader is referred to the text of [8], [13] and [19] for information about fixed-point index theory.

Given $A \subset B \subset N, \operatorname{cl}(A), \operatorname{cl}_{B}(A), \operatorname{int}(A), \operatorname{int}_{B}(A), \partial(A)$ and $\partial_{B}(A)$ will denote the closure of $A$, the closure of $A$ in $B$, the interior of $A$, the interior of $A$ in $B$, the boundary of $A$ and the boundary of $A$ in $B$ respectively.

We consider the exit set of $N$ to be defined as

$$
N^{-}=\{x \in N: f(x) \notin \operatorname{int}(N)\}
$$

The next definition is based on the notion of filtration introduced by Franks and Richeson in [15], and it is the key to the direct computation of the fixed-point index of any iteration of any homeomorphism of the plane. The reader can find the explicit definition of filtration pair in Appendix A.

Definition 1. Let $f: U \subset \mathbb{R}^{2} \rightarrow \mathbb{R}^{2}$ be a local homeomorphism. Suppose that $L \subset N$ is a compact pair contained in the interior of $U$. The pair $(N, L)$ is said to be a strong filtration pair for $f$ provided $N$ and $L$ are each the closure of their interiors and:

(1) $\quad N$ and $\partial(N \backslash L)$ are homeomorphic to a disk and $S^{1}$ respectively;

(2) $\operatorname{cl}(N \backslash L)$ is an isolating neighborhood;

(3) $\quad f(\operatorname{cl}(N \backslash L)) \subset \operatorname{int}(N)$ (i.e. $L$ is a neighborhood of $N^{-}$in $\left.N\right)$;

(4) for any component $L_{i}$ of $L, \partial_{N}\left(L_{i}\right)$ is an arc and there exists a topological disk $B_{i}$ such that $\partial_{N}\left(L_{i}\right) \subset B_{i} \subset L_{i}, B_{i} \cap N^{-} \neq \emptyset$ and $f\left(B_{i}\right) \cap \operatorname{cl}(N \backslash L)=\emptyset$.

THEOREM 1. (Baldwin and Slaminka) [1] Let $h: M \rightarrow M$ be an area-and orientationpreserving homeomorphism of an orientable 2-manifold $M$ having an isolated fixed point $p$ of index $-n$ for $n \geq 0$. Then there exists a topological disk $D$ with $p \in \operatorname{int}(D)$, $\operatorname{Fix}(h) \cap D=\{p\}$, having boundary $C$ with Lebesgue measure 0 , and there exist $2(n+1)$ compact, connected, area-zero sets $S_{0}, S_{1}, \ldots, S_{n}, U_{0}, U_{1}, \ldots, U_{n}$ which do not separate $M$ such that:

(1) $U_{i}, S_{i} \subset D$ and $U_{i}, S_{i}$ meet $C$ for all $i$;

(2) $p \in U_{i}, S_{i}$ for all $i$;

(3) $\quad h\left(S_{i}\right) \subset S_{i}, h^{-1}\left(U_{i}\right) \subset U_{i}$ for all $i$;

(4) $S_{i} \subset \Lambda^{+}(p)$ and $U_{i} \subset \Lambda^{-}(p)$ for all $i$;

(5) $S_{i} \cap S_{j}=\{p\}=U_{i} \cap U_{j}$ for all $i \neq j$;

(6) the sets $\left(S_{j} \cap C\right)$ and $\left(U_{i} \cap C\right)$ alternate on $C$.

The aim of this paper is to show that we can prove a general theorem for arbitrary homeomorphisms that admit a strong filtration in a neighborhood of an isolated fixed point. On the other hand the above theorem will become, using [9], a consequence of our results (see Remark 3). Let us begin with some intuitive examples.

Assume that $(N, L)$ is a strong filtration pair for a homeomorphism $f . L$ is the union of its components, $L=L_{1} \cup L_{2} \cup \cdots \cup L_{m}$. Let $K=\operatorname{Inv}(\operatorname{cl}(N \backslash L), f)$. 
We have that

$$
f \mid \operatorname{cl}(N \backslash L): \operatorname{cl}(N \backslash L) \rightarrow N
$$

and for every $i$, there exists $j$ such that $f\left(\partial_{N}\left(L_{i}\right)\right) \subset \operatorname{int}\left(L_{j}\right)$.

Consider the quotient space $D=\operatorname{cl}(N \backslash L) / \sim$ by identifying each $\partial_{N}\left(L_{i}\right)$ with a point $q_{i}$ (if $i \neq j$ then $q_{i} \neq q_{j}$ ).

Take the map projection

$$
\pi: \operatorname{cl}(N \backslash L) \rightarrow \operatorname{cl}(N \backslash L) / \sim
$$

and a retraction

$$
r: N \rightarrow \operatorname{cl}(N \backslash L)
$$

with $r(x)=x$ if $x \in \operatorname{cl}(N \backslash L)$ where $r$ retracts each $L_{i}$ to $\partial_{N}\left(L_{i}\right)$.

Now define $f^{\prime}=\pi \circ r \circ f \circ \pi^{-1}$,

$$
f^{\prime}: \operatorname{cl}(N \backslash L) / \sim \backslash\left\{q_{1}, \ldots, q_{m}\right\} \rightarrow \operatorname{cl}(N \backslash L) / \sim .
$$

Then $f^{\prime}$ is continuous and in a small enough neighborhood of $p, f^{\prime} \equiv f$. Since $f\left(\partial_{N}\left(L_{i}\right)\right) \subset \operatorname{int}\left(L_{j}\right), f^{\prime}$ admits a unique continuous extension

$$
\overline{f^{\prime}}: \operatorname{cl}(N \backslash L) / \sim \rightarrow \operatorname{cl}(N \backslash L) / \sim
$$

such that $\overline{f^{\prime}}\left(U^{\prime}\left(q_{i}\right)\right)=q_{j}$ for a neighborhood $U^{\prime}\left(q_{i}\right)$ of $q_{i}$.

$\overline{f^{\prime}}\left(\left\{q_{1}, \ldots, q_{m}\right\}\right) \subset\left\{q_{1}, \ldots, q_{m}\right\}$. In fact, $\overline{f^{\prime}}\left(q_{i}\right)=q_{j}$ if and only if $f\left(\partial_{N}\left(L_{i}\right)\right) \subset$ $\operatorname{int}\left(L_{j}\right)$.

Obviously

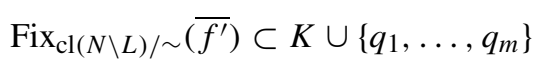

and since $K=\operatorname{Inv}(\operatorname{cl}(N \backslash L), f)$, it is clear that

$$
\operatorname{Fix}_{\mathrm{cl}(N \backslash L) / \sim\left(\left(\overline{f^{\prime}}\right)^{k}\right) \subset K \cup\left\{q_{1}, \ldots, q_{m}\right\} .}
$$

Examples. We will present some homeomorphisms and we will find the integers $r, q$ and $\delta$ of the above theorem.

Let $N=\left\{x \in \mathbb{R}^{2}:\|x\| \leq 2^{1 / 2}\right\}$ and let $f: \mathbb{R}^{2} \rightarrow \mathbb{R}^{2}$ be a homeomorphism generating the discrete dynamical system of Figure 1(a). Take $\epsilon>0$ big enough and let $L$ be the union of the $\epsilon$-neighborhoods, in $N$, of $(1,1),(-1,1),(-1,-1)$ and $(1,-1)$ (see Figure 1(b)).

Let $g, s: \mathbb{R}^{2} \rightarrow \mathbb{R}^{2}$ be a $\pi / 2$-rotation and a symmetry with respect to $\{x-y=0\}$ respectively.

We have that $0 \in N$ is a non-repeller rest point, $N$ is an isolating neighborhood of $\{0\}$ for $f, g \circ f$ and $s \circ f$. In all cases the pair $(N, L)$ is a strong filtration pair (for an adequate $\epsilon>0)$. Consider $q_{i}=\pi\left(L_{i}\right) \in \operatorname{cl}(N \backslash L) / \sim$ for $i \in\{1,2,3,4\}$.

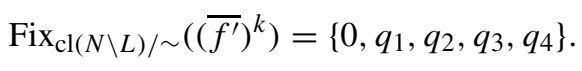

In this case we have, apart from 0 , four period-one periodic orbits so $r=1$ and $q=4$. Since $\overline{f^{\prime}}$ is constant in a neighborhood of each $q_{i}$ for $i \in\{1,2,3,4\}$ we have that for every $k \in \mathbb{N}$

$$
\begin{aligned}
& i_{\mathbb{R}^{2}}\left(f^{k}, 0\right)=1-4=-3,
\end{aligned}
$$

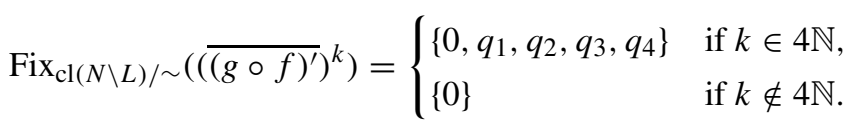




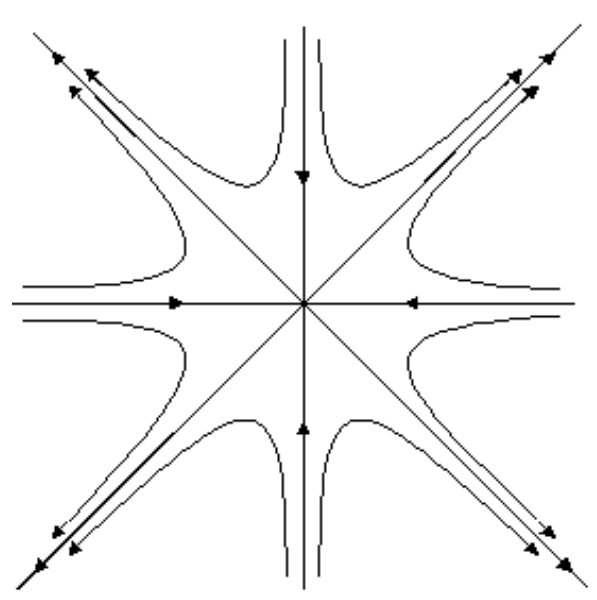

(a)

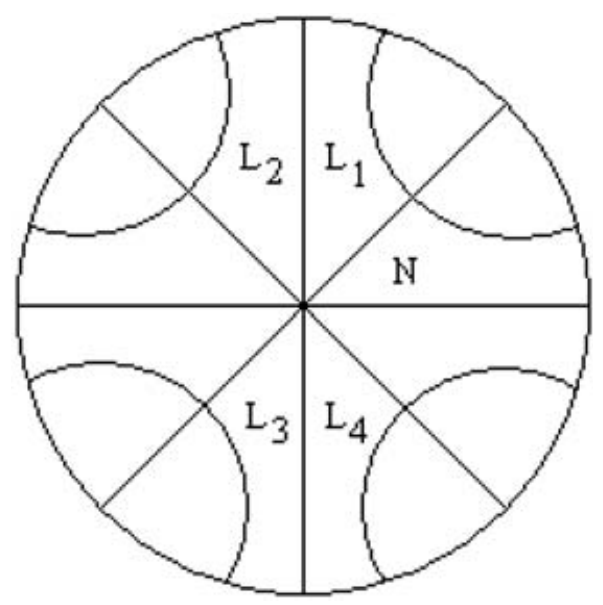

(b)

FIGURE 1. The homeomorphism $f$ and a strong filtration pair for $f$.

Now $\overline{(g \circ f)^{\prime}}$ has, apart from 0 , a period-four periodic orbit. So $r=4$ and $q=1$ and

$$
i_{\mathbb{R}^{2}}\left((g \circ f)^{k}, 0\right)= \begin{cases}-3 & \text { if } k \in 4 \mathbb{N}, \\ 1 & \text { if } k \notin 4 \mathbb{N} .\end{cases}
$$

On the other hand, $s \circ f$ is orientation-reversing:

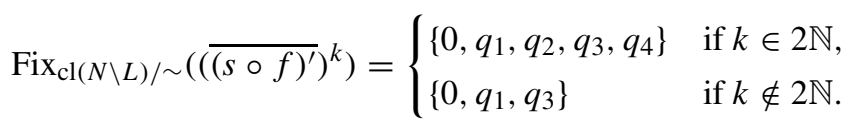

$\overline{(s \circ f)^{\prime}}$ has, apart from $0, q=1$ periodic orbits of period two and two fixed points. So,

$$
i_{\mathbb{R}^{2}}\left((s \circ f)^{k}, 0\right)= \begin{cases}-3 & \text { if } k \text { even } \\ -1 & \text { if } k \text { odd. }\end{cases}
$$

The main result of this paper is that phase spaces as in the examples are in some way canonical models for all similar local homeomorphisms with the same associated integers $r$ and $q$ ( $\delta$ and $q$ ) if $f$ preserves the orientation (if $f$ reverses the orientation). The reader can easily check that for the above maps there exist branches of the stable and unstable manifolds as in Theorem 1, even though the involved maps do not preserve area or orientation or have index $<1$. The reason is that all of them admit strong filtration pairs about $p$.

The paper is organized as follows. In $\S 2$ we give a theorem that computes the fixed-point index of every iteration of a homeomorphism $f$ in a neighborhood of an isolated compact invariant set that admits a strong filtration pair and we prove some preparatory results. Section 3 is devoted to proving the Main Theorem and to stating its consequences. The most important one, Corollary 5, describes the stable and unstable manifolds in a neighborhood of a fixed point that is a compact isolated invariant set which is 
neither an attractor nor a repeller. Appendix A contains the proof of a theorem of existence of strong filtration pairs (Theorem 3). This theorem is an extension of Theorem 1 in [21]. For the sake of completeness we decided to include it here as an appendix.

\section{Preliminary results}

Given a homeomorphism $f: U \subset \mathbb{R}^{2} \rightarrow f(U) \subset \mathbb{R}^{2}$, let us assume that we have a strong filtration pair $(N, L)$ and let $\overline{f^{\prime}}: \operatorname{cl}(N \backslash L) / \sim \rightarrow \operatorname{cl}(N \backslash L) / \sim$ be the induced map. We maintain the notation of the introduction.

Definition 2. Let $\theta=\left\{p_{1}, \ldots, p_{s}\right\} \subset\left\{q_{1}, \ldots, q_{m}\right\}$ be a subset on which $\overline{f^{\prime}}$ acts as a permutation. We say that $p_{i}, p_{j} \in \theta$ are adjacent if there is an $\operatorname{arc} \gamma \subset \partial(N \backslash L) / \sim$ joining $p_{i}$ and $p_{j}$ such that $\gamma \cap \theta=\left\{p_{i}, p_{j}\right\}$.

The next proposition says that $\overline{f^{\prime}}$ preserves adjacency in every subset $\theta$ on which it acts as a permutation. Even though it is stated in a more general setting than that of [21] the proof needs only minor changes.

Proposition 1. [21] Let $\theta=\left\{p_{1}, \ldots, p_{s}\right\} \subset\left\{q_{1}, \ldots, q_{m}\right\}$ be a subset such that $\overline{f^{\prime}}(\theta)=\theta$. If $p_{i}$ and $p_{j}$ are adjacent in $\theta$, then their images $p_{i+1}$ and $p_{j+1}$ are also adjacent.

COROLLARY 1. [21] Under the above conditions:

(a) if $f$ is orientation-preserving then all the periodic orbits of $\overline{f^{\prime}}$, in $\left\{q_{1}, \ldots, q_{m}\right\}$, have the same period;

(b) if $f$ is orientation-reversing then $\overline{f^{\prime}}$ has no more than two fixed points in $\left\{q_{1}, \ldots, q_{m}\right\}$ and the period of its periodic points is $\leq 2$.

Using Corollary 1, we have the following theorem.

THEOREM 2. Let $f: U \subset \mathbb{R}^{2} \rightarrow f(U) \subset \mathbb{R}^{2}$ be a homeomorphism. Suppose that there exists a strong filtration pair, $(N, L)$, for $f$ and let $K=\operatorname{Inv}(\operatorname{cl}(N \backslash L), f)$. Then, there are an AR (absolute retract for metric spaces), $D$, containing a neighborhood $V \subset \mathbb{R}^{2}$ of $K$, a finite subset $\left\{q_{1}, \ldots, q_{m}\right\} \subset D$ and a map $\overline{f^{\prime}}: D \rightarrow D$ such that $\left.\overline{f^{\prime}}\right|_{V}=\left.f\right|_{V}$ and for every $k \in \mathbb{N}, \operatorname{Fix}\left(\left(\overline{f^{\prime}}\right)^{k}\right) \subset K \cup\left\{q_{1}, \ldots, q_{m}\right\}$.

Moreover:

(a) if $f$ preserves the orientation, then

$$
i_{\mathbb{R}^{2}}\left(f^{k}, K\right)= \begin{cases}1-r q & \text { if } k \in r \mathbb{N}, \\ 1 & \text { if } k \notin r \mathbb{N},\end{cases}
$$

where $k \in \mathbb{N}, q$ is the number of periodic orbits of $\overline{f^{\prime}}$ in $\left\{q_{1}, \ldots, q_{m}\right\}$ and $r$ is their period;

(b) if $f$ reverses the orientation, then

$$
i_{\mathbb{R}^{2}}\left(f^{k}, K\right)= \begin{cases}1-\delta & \text { if } k \text { odd }, \\ 1-\delta-2 q & \text { if } k \text { even },\end{cases}
$$

where $\delta \in\{0,1,2\}$ and $q$ are the number of fixed points and period-two orbits of $\overline{f^{\prime}}$ in $\left\{q_{1}, \ldots, q_{m}\right\}$, respectively. 
Definition 3. Under the setting of the above theorem, the integer $r$ ( $r=2$ if $f$ is orientation reversing) is called the period of the strong filtration pair $(N, L)$.

The next theorem is a generalization of Theorem 1 in [21]. In order to keep this paper as self-contained as possible we will give the details of the proof in Appendix A.

THEOREM 3. Let $f: U \subset \mathbb{R}^{2} \rightarrow f(U) \subset \mathbb{R}^{2}$ be a homeomorphism. Suppose that there is a topological disk $D \subset U$ such that $\operatorname{Fix}(f) \cap D=\{p\}, \operatorname{Inv}(D, f) \subset \operatorname{int}(D)$ and that there is not a disk $B \subset D$ containing $p$ such that $B \subset \operatorname{int}(f(B))$. Then, there is a strong filtration pair $(N, L)$ with $p \in K=\operatorname{Inv}(\operatorname{cl}(N \backslash L), f)$.

Let us recall the following result due to Le Calvez [9]. This theorem is a local version of Brouwer's lemma on translation arcs. The theorem essentially says that, for orientationpreserving homeomorphisms, the existence of a recurrent orbit implies that the index of the fixed point is 1 .

THEOREM 4. Let $f: W \rightarrow W^{\prime}$ be an orientation-preserving homeomorphism between two simply connected neighborhoods of $0 \in \mathbb{R}^{2}$ such that $\operatorname{Fix}(f)=\{0\}$. Let $W^{\prime \prime}$ be the connected component of 0 in $W \cap f^{-1}(W)$. If there is a domain $V \subset W^{\prime \prime}$ such that $f(V) \cap V=\emptyset$, a point $z \in V$ and an integer $q \geq 2$ such that $f^{i}(z) \in W^{\prime \prime}$ for every $i \in\{1, \ldots, q-1\}$ and $f^{q}(z) \in V$, then $i_{\mathbb{R}^{2}}(f, 0)=1$.

Some known consequences of Theorems 2, 3 and 4 are listed in the following remark.

Remark 1. Let $f: U \subset \mathbb{R}^{2} \rightarrow f(U) \subset \mathbb{R}^{2}$ be a homeomorphism and let $p \in U$ be a fixed point of $f$. If there is a topological disk $D$ such that $\operatorname{Fix}(f) \cap D=\{p\}$ and $\operatorname{Inv}(D, f) \subset \operatorname{int}(D)$, it follows that $i_{\mathbb{R}^{2}}(f, p) \leq 1$. Moreover, if $\operatorname{Fix}\left(f^{s}\right) \cap D=\{p\}$ then $i_{\mathbb{R}^{2}}\left(f^{s}, p\right) \leq 1$. Indeed, if there is a disk $B \subset D$ containing $p$ such that $B \subset \operatorname{int}(f(B))$, then $\left|i_{\mathbb{R}^{2}}\left(f^{-1}, p\right)\right|=\left|i_{\mathbb{R}^{2}}(f, p)\right|=1$. In either case we apply Theorem 3 and Theorem 2 .

On the other hand, if $i_{\mathbb{R}^{2}}(f, p)>1$, then $\operatorname{Inv}(D, f) \cap \partial(D) \neq \varnothing$ for every small enough disk $D$ such that $p \in \operatorname{int}(D)$. In particular, there are full orbits $\gamma \neq p$ in every neighborhood of $p$. Moreover, if $f$ preserves the orientation, using Theorem 4 it follows that $\lim _{k \rightarrow \infty} f^{k}(x)=p$ (respectively $\lim _{k \rightarrow \infty} f^{-k}(x)=p$ ) for every $x \in \operatorname{Inv}^{+}(D, f)$ (respectively for every $x \in \operatorname{Inv}^{-}(D, f)$ ).

For a special class of non-saddle fixed points we have a sort of converse of the last remark.

COROllaRY 2. Let $U \subset \mathbb{R}^{2}$ be an open ball and let $f: U \rightarrow U$ be a homeomorphism such that $\operatorname{Fix}(f)=\{p\}$. If $\Lambda^{+}(p) \cup \Lambda^{-}(p)=U$ and for every topological disk $B$ containing $p, \Lambda^{+}(p) \cap(U \backslash B) \neq \emptyset$ and $\Lambda^{-}(p) \cap(U \backslash B) \neq \emptyset$, then there is $r>1$ such that $i_{\mathbb{R}^{2}}\left(f^{r}, p\right)>1$. In particular, $\operatorname{Inv}(D, f) \cap \partial(D) \neq \emptyset$ for every small enough disk $D$ such that $p \in \operatorname{int}(D)$ and there are full orbits $\gamma \neq p$ in every neighborhood of $p$.

Proof. The sphere $S^{2}=U \cup\{q\}$ is the one-point compactification of $U$. Denote again by $f$ the obvious extension of $f$ to $S^{2}$. It is clear that $q$ is an isolated fixed point of $f$ such that $\{q\}$ is an isolated invariant set which is neither an attractor nor a repeller. Then $\{q\}$ admits a strong filtration pair and there is $r>1$ such that $i_{\mathbb{R}^{2}}\left(f^{r}, q\right)=1-s<1$. 
Consequently, since $f^{r}$ is orientation-preserving, $i_{\mathbb{R}^{2}}\left(f^{r}, p\right)=1+s>1$. The remaining part of the proof follows from Remark 1.

The next proposition is based on Theorem 4. However, note that we do not assume $f$ to be orientation-preserving.

PROPOSITION 2. Let $U \subset \mathbb{R}^{2}$ be an open ball and let $f: U \subset \mathbb{R}^{2} \rightarrow f(U) \subset \mathbb{R}^{2}$ be a homeomorphism. Let $p \in U$ be a fixed point of $f$. Suppose that there is a strong filtration pair of period $r,(N, L)$, such that $p \in \operatorname{Inv}(\operatorname{cl}(N \backslash L), f), f^{j}(\operatorname{cl}(N \backslash L)) \subset U$ for any $j \in\{1, \ldots, r\}$ and $\operatorname{Fix}\left(f^{r}\right) \cap \operatorname{cl}(N \backslash L)=\{p\}$. Then, $\lim _{k \rightarrow \infty} f^{k}(x)=p$, for every $x \in K^{+}=\operatorname{Inv}^{+}(\operatorname{cl}(N \backslash L), f)$.

Proof. Note that $f^{r}$ is orientation-preserving. Also, $\operatorname{cl}(N \backslash L)$ is a connected subset of $U \cap f^{-1}(U)$.

Given $x \in K^{+}$, we will show first that $\lim _{k \rightarrow \infty} f^{k r}(x)=p$.

We will adapt the arguments of the proof of the above theorem of Le Calvez [9] to our situation. Let $D$ be a disk such that $\bigcup_{j \in\{1, \ldots, r\}} f^{j}(\operatorname{cl}(N \backslash L)) \subset \operatorname{int}(D) \subset D \subset U$.

The restriction of $f$ to $D$ can be extended to a homeomorphism, which we shall denote again by $f, f: \mathbb{R}^{2} \rightarrow \mathbb{R}^{2}$ (see [11] for example). Let $R=\left\{x \in \mathbb{R}^{2} \backslash\{p\}: f^{r}(x)=x\right\}$ and let $M_{p}$ be the component of $\mathbb{R}^{2} \backslash R$ containing $p$. Then $\operatorname{cl}(N \backslash L) \subset M_{p}$. Let $\pi: \overline{M_{p}} \rightarrow M_{p}$ be the universal covering of $M_{p}$. It is well known that $\overline{M_{p}}$ is again a plane. Fix $z_{0} \in \pi^{-1}(p)$ and let $F: \overline{M_{p}} \rightarrow \overline{M_{p}}$ be the lift of $f \mid M_{p}$ such that $F\left(z_{0}\right)=z_{0}$. Then, the set of period- $r$ periodic points of $F$ is a non-empty (and discrete) subset of $\pi^{-1}(p)$. Now, $F^{r}$ is conjugate to $f^{r}$ in a neighborhood of each period- $r$ periodic point.

Take $\epsilon>0 . K^{+} \backslash B(p, \epsilon)$ is a compact subset of $U$ and for every $y \in K^{+} \backslash B(p, \epsilon)$ there is $\delta_{y}$ such that $f^{r}\left(B\left(y, \delta_{y}\right)\right) \cap B\left(y, \delta_{y}\right)=\emptyset$. Then, $K^{+} \backslash B(p, \epsilon) \subset \bigcup_{y \in K^{+} \backslash B(p, \epsilon)} K^{+} \cap$ $B\left(y, \delta_{y} / 2\right)$ and there is a finite subset $P=\left\{y_{1}, \ldots, y_{s}\right\}$ such that $K^{+} \backslash B(p, \epsilon) \subset$ $\bigcup_{j \in\{1, \ldots, s\}} K^{+} \cap B\left(y_{j}, \delta_{y_{j}} / 2\right)$.

If there is an increasing sequence $\left\{k_{n}\right\} \rightarrow \infty$ such that $f^{k_{n} r}(x) \notin B(p, \epsilon)$ there is $y_{i} \in P$ and a subsequence $\left\{r_{n}\right\} \subset\left\{k_{n}\right\}$ such that $f^{r_{n} r}(x) \in K^{+} \cap B\left(y_{i}, \delta_{y_{i}} / 2\right)$. Take $z=f^{r_{1} r}(x), q=r_{k}-r_{1} \geq 2$ for a big enough $k$, and $V=B\left(y_{i}, \delta_{y_{i}} / 2\right)$. Then, $f^{r}(V) \cap V=\emptyset,\left\{f^{r}(z), \ldots, f^{r(q-1)}(z)\right\} \subset K^{+}$and $f^{r q}(z) \in V$.

Let $\bar{D}$ be the component of $\pi^{-1}(D)$ containing $z_{0}$ and $\bar{V}$ be the component of $\pi^{-1}(V)$ contained in $\bar{D}$. Then, we have that $F^{r}(\bar{V}) \cap \bar{V}=\emptyset$ and $F^{r q}(\bar{V}) \cap \bar{V} \neq \emptyset$. Since $i_{\mathbb{R}^{2}}\left(f^{r}, p\right)=i_{\mathbb{R}^{2}}\left(F^{r}, z_{0}\right)<1$ we get a contradiction to Theorem 4 .

Now consider $x \in K^{+}$and suppose that there is a sequence $\left\{k_{n}\right\} \rightarrow \infty$ such that $\lim _{n \rightarrow \infty} f^{k_{n}}(x)=q \neq p$. We can assume that there is $l$ such that $k_{n} \equiv l \bmod r$. Then, we have that for $x^{\prime}=f^{l}(x) \in K^{+}$there is a sequence $m_{n}$ such that $\lim _{n \rightarrow \infty} f^{m_{n} r}\left(x^{\prime}\right)=q$. Therefore, $q=p$.

Proposition 3. Let $f: U \subset \mathbb{R}^{2} \rightarrow f(U) \subset \mathbb{R}^{2}$ be a homeomorphism. Let $D \subset U$ be a disk and let $K^{+}=\operatorname{Inv}^{+}(D, f)$ (respectively $K^{-}=\operatorname{Inv}^{-}(D, f)$ ). Then, every component of $K^{+}$(respectively $K^{-}$) is a trivial shape continuит. 
Proof. Take a component $C$ of $K^{+}$. If $C$ is a non-trivial shape continuum, using Borsuk's shape classification of planar continua [3] it follows that $\mathbb{R}^{2} \backslash C$ is not connected. Let $V$ be a bounded component of $\mathbb{R}^{2} \backslash C$. Then $\partial(V) \subset C \subset K^{+} \subset D$ and there is a component $C_{1}$ of $K^{+}$such that $\partial f(V) \subset C_{1} \subset K^{+} \subset D$. Then $f(V) \subset D$. Now, by an induction argument we have that $f^{k}(V) \subset D$ for every $k \in \mathbb{N}$. Therefore $V \subset K^{+}$and we get a contradiction.

Remark 2. Under the setting of Proposition 2 we have that the components of $K^{+}$and $K^{-}$ are trivial shape continua such that $\lim _{k \rightarrow \infty} f^{k}(x)=p$ for $x \in K^{+}=\operatorname{Inv}^{+}(\operatorname{cl}(N \backslash L), f)$ and $\lim _{k \rightarrow \infty} f^{-k}(x)=p$ for $x \in K^{-}=\operatorname{Inv}^{-}(\operatorname{cl}(N \backslash L), f)$.

\section{The Main Theorem and its consequences}

Let $f: U \subset \mathbb{R}^{2} \rightarrow f(U) \subset \mathbb{R}^{2}$ be a homeomorphism and let $(N, L)$ be a strong filtration pair of period $r$. We denote $N_{L}=\operatorname{cl}(N \backslash L) / \sim$.

Proposition 4. $\operatorname{Inv}^{-}\left(N_{L}, \overline{f^{\prime}}\right)=\bigcap_{n \in \mathbb{N}}\left(\overline{f^{\prime}}\right)^{n}\left(N_{L}\right)$. Then $\operatorname{Inv}^{-}\left(N_{L}, \overline{f^{\prime}}\right)$ is a continuum.

Proposition 5. $\operatorname{Inv}^{-}(\operatorname{cl}(N \backslash L), f) / \sim=\operatorname{Inv}^{-}\left(N_{L}, \overline{f^{\prime}}\right)$.

Proof. Let $[x] \in \operatorname{Inv}^{-}(\operatorname{cl}(N \backslash L), f) / \sim \subset N_{L}$. We have that there exists a negative semiorbit of $x$ (with respect to $f$ ), $\gamma^{-}(x)$, such that $\left(\gamma^{-}(x) \backslash\{x\}\right) \cap\left(\bigcup \partial_{N}\left(L_{i}\right)\right)=\emptyset$. Then it is clear that $[x] \in \operatorname{Inv}^{-}\left(N_{L}, \overline{f^{\prime}}\right)$.

On the other hand, for $[x] \in \operatorname{Inv}^{-}\left(N_{L}, \overline{f^{\prime}}\right)$ with $[x] \notin\left\{q_{1}, \ldots, q_{m}\right\}$, then the negative semiorbit of $[x]$ (with respect to $\left.\overline{f^{\prime}}\right), \gamma^{-}([x])$, is such that $\gamma^{-}([x]) \cap\left\{q_{1}, \ldots, q_{m}\right\}=\emptyset$. Then it is clear that $[x] \in \operatorname{Inv}^{-}(\operatorname{cl}(N \backslash L), f) / \sim$.

Let us consider the set

$$
A=\operatorname{Inv}^{-}\left(N_{L}, \overline{f^{\prime}}\right) \backslash\left\{q_{1}, \ldots, q_{m}\right\} \subset \operatorname{Inv}^{-}(\operatorname{cl}(N \backslash L), f) / \sim .
$$

Since $\operatorname{Inv}^{-}\left(N_{L}, \overline{f^{\prime}}\right)$ is a continuum we have

$$
\operatorname{cl}(A)=\operatorname{Inv}^{-}\left(N_{L}, \overline{f^{\prime}}\right) \subset \operatorname{Inv}^{-}(\operatorname{cl}(N \backslash L), f) / \sim
$$

and the result is proved.

MAIN TheOREM. Let $f: U \subset \mathbb{R}^{2} \rightarrow f(U) \subset \mathbb{R}^{2}$ be a homeomorphism. Let $p$ be an isolated fixed point of $f$ and let us assume that there is a strong filtration pair of period $r$, $(N, L)$, such that $p \in \operatorname{int}(N \backslash L), L \neq \emptyset, f^{j}(\operatorname{cl}(N \backslash L)) \subset U$ for any $j \in\{1, \ldots, r\}$ and $\operatorname{Fix}\left(f^{r}\right) \cap \operatorname{cl}(N \backslash L)=\{p\}$. Let $H_{p}^{+}, H_{p}^{-}$be the components of $K^{+}$and $K^{-}$respectively containing $p$. Then, $H_{p}^{+}$and $H_{p}^{-}$are trivial shape continua such that $\lim _{k \rightarrow \infty} f^{k}(x)=p$ for every $x \in H_{p}^{+}$and $\lim _{k \rightarrow \infty} f^{-k}(x)=p$ for every $x \in H_{p}^{-}$. The sets $H_{p}^{+} \cap \partial(\operatorname{cl}(N \backslash L))$ and $H_{p}^{-} \cap \partial(\operatorname{cl}(N \backslash L))$ have at least $1-i_{\mathbb{R}^{2}}\left(f^{r}, p\right)$ components.

Proof. Let us study the set $H_{p}^{+}$. Using Propositions 3 and 2 we have that $H_{p}^{+}$is a trivial shape continuum such that $\lim _{k \rightarrow \infty} f^{k}(x)=p$ for every $x \in H_{p}^{+}$. Only a proof of the fact that $H_{p}^{+} \cap \partial(\operatorname{cl}(N \backslash L))$ has at least $1-i_{\mathbb{R}^{2}}\left(f^{r}, p\right)$ components is needed.

We will keep the notation of Theorem 2. Let us write again $N_{L}=\operatorname{cl}(N \backslash L) / \sim$. When it is clear from the context we will denote $Q \subset N \backslash L$ and $\pi(Q) \subset N_{L}$ 
in the same way. Let $\overline{f^{\prime}}: N_{L} \rightarrow N_{L}$ be the map induced by $f$ and let $\theta=\left\{p_{1}, \ldots, p_{s}\right\}$ be the biggest subset of $\left\{q_{1}, \ldots, q_{m}\right\}$ on which $\overline{f^{\prime}}$ acts as a permutation. From Theorem 2 ,

$$
i_{\mathbb{R}^{2}}\left(f^{r}, K\right)=i_{\mathbb{R}^{2}}\left(f^{r}, p\right)=1-s .
$$

It is clear that $\theta$ is an attractor for $\overline{f^{\prime}}$. In fact, every $p_{i} \in \theta$ has a neighborhood $U^{\prime}\left(p_{i}\right)$ such that $\overline{f^{\prime}}\left(U^{\prime}\left(p_{i}\right)\right)=p_{j}$.

Let $A$ be the region of attraction of $\theta$ :

$$
A=\left\{x \in N_{L}: \text { there is } n_{0} \text { such that }\left(\overline{f^{\prime}}\right)^{n_{0}}(x) \in \theta\right\} .
$$

First, let us show that $K^{+}=\operatorname{Inv}^{+}(\operatorname{cl}(N \backslash L), f)=N_{L} \backslash A$. Indeed, if $x \in N_{L} \backslash A$ then $\left(\overline{f^{\prime}}\right)^{n}(x) \notin \theta$ for any $n \in \mathbb{N}$ and consequently $f^{n}(x) \in \operatorname{int}_{N}(\operatorname{cl}(N \backslash L))$ for every $n \geq 0$. On the other hand, given $x \in K^{+}, f^{n}(x) \in \operatorname{cl}(N \backslash L)$ for every $n \in \mathbb{N}$. Then, using condition (4) in the definition of strong filtration pairs, $f^{n}(x) \in \operatorname{int}_{N}(\operatorname{cl}(N \backslash L))$ for every $n \geq 0$ and $\left(\overline{f^{\prime}}\right)^{n}(x)$ never belongs to $\theta$.

We will discuss separately the cases $s=1$ and $s>1$.

Assume that $s=1$. This case is classical and goes back to Birkhoff in the study of the regions of instability. However, the techniques we use to prove the theorem in this particular situation will lead us to a proof of the general case. If $s=1$ then $r=1$ and $i_{\mathbb{R}^{2}}(f, p)=0$. We have to check that $H_{p}^{+} \cap \partial(\operatorname{cl}(N \backslash L)) \neq \emptyset$. Suppose that $H_{p}^{+} \cap \partial(\operatorname{cl}(N \backslash L))=\emptyset$. Take an open ball $B$ such that $H_{p}^{+} \subset B \subset \operatorname{cl}(B) \subset \operatorname{int}(\operatorname{cl}(N \backslash L))$.

Let $\square\left(K^{+}\right)$be the space of components of $K^{+}$and let $P: K^{+} \rightarrow \square\left(K^{+}\right)$be the natural projection. It is well known, see [3] and [4] for example, that $\square\left(K^{+}\right)$is a zerodimensional compactum. $P\left(K^{+} \backslash B\right)$ is a compact subset of $\square\left(K^{+}\right)$; then there is a clopen (open and closed) neighborhood, $V$, of $P\left(H_{p}^{+}\right)$such that $V \cap P\left(K^{+} \backslash B\right)=\emptyset$. Then, $P^{-1}(V)$ is a clopen neighborhood of $H_{p}^{+}$in $K^{+}$and it is union of components of $K^{+}$contained in $B$. There is an open subset $U \subset B$ of $\operatorname{int}(\operatorname{cl}(N \backslash L))$ such that $U \cap K^{+}=\operatorname{cl}(U) \cap K^{+}=P^{-1}(V)$. Now let $\alpha: B \rightarrow \mathbb{R}^{+}$be a smooth function which vanishes exactly on $\operatorname{cl}(U)$. For any regular value $\epsilon>0, \alpha^{-1}([0, \epsilon])$ is a compact manifold with boundary. Taking a small enough regular value $\epsilon$, we have that $\alpha^{-1}([0, \epsilon])$ is a manifold such that $\alpha^{-1}([0, \epsilon]) \cap K^{+}=P^{-1}(V)$. Then $\alpha^{-1}([0, \epsilon])$ is a topological disk with a finite number of holes and we can fill these holes to obtain a disk $D$ such that $H_{p}^{+} \subset \operatorname{int}(D) \subset D \subset \operatorname{int}(\operatorname{cl}(N \backslash L))$ and $\partial(D) \cap K^{+}=\emptyset$. Since $\partial(D)$ is a compact subset of $A$, there exists $n_{0}>0$ such that $\left(\overline{f^{\prime}}\right)^{n_{0}}(\partial(D)) \subset \theta$. Now consider $f(\partial(D))$. If $f(\partial(D)) \cap L \neq \emptyset$ we can take another disk $D_{1}$ such that $p \in D_{1}, \partial\left(D_{1}\right) \cap K^{+}=\emptyset$ and $\partial\left(D_{1}\right) \subset f(\partial(D)) \cup \partial_{N}(L)$. If $f(\partial(D)) \cap L=\emptyset$ we take $D_{1}=f(D)$. By an elemental induction argument, we can obtain a disk, $D_{n_{0}-1}$, containing $p$, such that $\partial\left(D_{n_{0}-1}\right) \subset f^{n_{0}-1}(\partial(D)) \cup \partial_{N}(L)$ and $f\left(\partial\left(D_{n_{0}-1}\right)\right) \subset L$. Then $p \in L$ and we have a contradiction.

Now suppose that $s>1 . \beta=\left(\bar{f}^{\prime}\right)^{r}$ fixes every element $p_{j} \in \theta, j \in\{1, \ldots, s\}$. Let $A\left(p_{j}\right)$ be the component of $A$ containing $p_{j}$. It is obvious that $K^{+}$separates the components $A\left(p_{j}\right), j \in\{1, \ldots, s\}$. For every element $p_{j} \in \theta$ there is a component of $\partial\left(A\left(p_{j}\right)\right) \subset K^{+}$separating $p_{j}$ from $\theta \backslash p_{j}$. Indeed, in either case, using similar arguments to that of the above paragraph, one can obtain a finite family of mutually disjoint topological disks $D_{1}, \ldots, D_{l}$, contained in $N \backslash L$ and covering $\partial\left(A\left(p_{j}\right)\right)$, such that 
$\partial\left(A\left(p_{j}\right)\right) \cap \partial_{\mathrm{cl}(N \backslash L)}\left(D_{i}\right)=\emptyset$ for every $i \in\{1, \ldots, l\}$ and none of them separates $p_{j}$ from $\theta \backslash p_{j}$. Then one could join $p_{j}$ with $\theta \backslash p_{j}$ by a path that does not intersect $\partial\left(A\left(p_{j}\right)\right)$, and this is not possible.

Let $K_{p_{j}}$ be the component of $\partial\left(A\left(p_{j}\right)\right)$ separating $p_{j}$ from $\theta \backslash p_{j}$. It is clear that $\beta\left(\partial\left(A\left(p_{j}\right)\right)\right)=f^{r}\left(\partial\left(A\left(p_{j}\right)\right)\right) \subset \partial\left(A\left(p_{j}\right)\right)$; then from Proposition $2, p \in K_{p_{j}}$. Consequently $K_{p_{j}} \subset H_{p}^{+}$.

We have that $H_{p}^{+}$intersects at least twice $\partial(\operatorname{cl}(N \backslash L))$ for every element of $\theta$. Now the result follows immediately.

By Propositions 2 and $3, H_{p}^{-}$is a trivial shape continuum such that $\lim _{k \rightarrow \infty} f^{-k}(x)=p$ for every $x \in H_{p}^{-}$and, by Corollary $3, H_{p}^{-} \cap \partial(\operatorname{cl}(N \backslash L))$ has at least $1-i_{\mathbb{R}^{2}}\left(f^{r}, p\right)=s$ components $\left(H_{p}^{-} \cap \partial_{N}\left(L_{i}\right) \neq \emptyset\right.$ for every $\left.i=1, \ldots, s\right)$.

COROLlaRY 3. Let $(N, L)$ be a strong filtration pair of period $r,\{p\}=\operatorname{Inv}(\operatorname{cl}(N \backslash L), f)$ and $\theta=\left\{p_{1}, \ldots, p_{s}\right\} \subset\left\{q_{1}, \ldots, q_{m}\right\}$ be the family of periodic points of $\overline{f^{\prime}}$ in $\left\{q_{1}, \ldots, q_{m}\right\} . \quad$ It is clear that $\{p\} \cup \theta \subset \operatorname{Inv}^{-}(\operatorname{cl}(N \backslash L), f) / \sim$. The component of $K^{-}=\operatorname{Inv}^{-}(\operatorname{cl}(N \backslash L), f)$ containing $p, H_{p}^{-}$, is such that $H_{p}^{-} \cap \partial(\operatorname{cl}(N \backslash L))$ has at least $1-i_{\mathbb{R}^{2}}\left(f^{r}, p\right)=s$ components $\left(\partial_{N}\left(L_{i}\right) \cap H_{p}^{-} \neq \emptyset\right.$ for all $\left.i=1, \ldots, s\right)$.

Proof. We maintain the notation of the proof of the Main Theorem. Let us define $K_{i}=\bigcap_{n \in \mathbb{N}}\left(\overline{f^{\prime}}\right)^{n r}\left(\operatorname{cl}\left(A\left(p_{i}\right)\right)\right)$ for every $i \in\{1, \ldots, s\}$.

Since $\left.\overline{\left(f^{\prime}\right.}\right)^{r}\left(\operatorname{cl}\left(A\left(p_{i}\right)\right)\right) \subset \operatorname{cl}\left(A\left(p_{i}\right)\right)$, it is clear that $K_{i}$ is a continuum with $\left\{p, p_{i}\right\} \subset$ $K_{i}=\left(\overline{f^{\prime}}\right)^{r}\left(K_{i}\right) \subset \operatorname{cl}\left(A\left(p_{i}\right)\right)$. Then the corollary follows from the fact that $\bigcup_{i \in\{1, \ldots, s\}}$ $\left(K_{i} \backslash\left\{p_{i}\right\}\right) \subset H_{p}^{-}$.

Proposition 6. Let $f: U \subset \mathbb{R}^{2} \rightarrow f(U) \subset \mathbb{R}^{2}$ be a homeomorphism and let $p$ be a fixed point of $f$ such that $\{p\}$ is an isolated invariant set which is neither an attractor nor a repeller. Let us consider a strong filtration pair $(N, L)$ of period $r$ such that $\{p\}=\operatorname{Inv}(\operatorname{cl}(N \backslash L), f)$. Then, the component $H_{p}^{+}$of $K^{+}$containing $p$ is such that the number of components of $H_{p}^{+} \backslash\{p\}$ is $\geq 1-i_{\mathbb{R}^{2}}\left(f^{r}, p\right)$. If there is a component of $H_{p}^{+} \backslash\{p\}$ which does not intersect $\partial(\operatorname{cl}(N \backslash L))$ then both the number of components which do not intersect $\partial(\operatorname{cl}(N \backslash L))$ and the number of components which intersect $\partial(\operatorname{cl}(N \backslash L))$ are infinite.

In the proof of this proposition we need the following lemma.

LEMMA 1. Let $B$ be a continuum and let $A$ be a proper subcontinuum of $B$. Then for every closed neighborhood $V$ of $A$ in $B$ there exists a continuum $\gamma \neq A$ such that $A \subset \gamma \subset V$.

Proof. Assume that there is $V$ such that $A$ is a component of $V$. Using that $\square(V)$ is zero-dimensional it is easy to check that $B$ is not connected.

Proof of Proposition 6. Let $\theta=\left\{p_{1}, \ldots, p_{s}\right\}$ be the set of periodic points of $\overline{f^{\prime}}$ in $\left\{q_{1}, \ldots, q_{m}\right\} \subset N_{L}$. We have that $\theta \subset H_{p}^{-} / \sim$ and $H_{p}^{-} / \sim \bigcap\left(H_{p}^{+} \backslash\{p\}\right)=\emptyset$. Since $\left(H_{p}^{+} \backslash\{p\}\right) \cap \partial(\operatorname{cl}(N \backslash L))=H_{p}^{+} \cap \partial(\operatorname{cl}(N \backslash L))$ then the set $H_{p}^{+} \backslash\{p\}$ has at least $s=1-i_{\mathbb{R}^{2}}\left(f^{r}, p\right)$ components (see the proof of the Main Theorem) which are separated by the continuum $H_{p}^{-} / \sim$.

Let us denote $H_{p}^{+} \backslash\{p\}=\bigcup \gamma_{k}^{+}$, where each $\gamma_{k}^{+}$is a component of $H_{p}^{+} \backslash\{p\}$. 
We have that $\gamma_{k}^{+} \cup\{p\}=\operatorname{cl}\left(\gamma_{k}^{+}\right)$. Indeed, $\gamma_{k}^{+}$is closed in $H_{p}^{+} \backslash\{p\}$, then $\operatorname{cl}_{H_{p}^{+}}\left(\gamma_{k}^{+}\right)$is $\gamma_{k}^{+}$or $\gamma_{k}^{+} \cup\{p\}$. In the first case, using the above lemma, we have a contradiction. On the other hand, it is not difficult to prove that each $\operatorname{cl}\left(\gamma_{k}^{+}\right)$has trivial shape (see Proposition 3).

Take $\gamma_{k}^{+}$, then $\overline{f^{\prime}}\left(\gamma_{k}^{+}\right) \subset \gamma_{k^{\prime}}^{+}$. On the other hand, since $f$ is a homeomorphism and $f=\overline{f^{\prime}}$ in a neighborhood of $p$, there exists a $\gamma_{k^{\prime \prime}}^{+}$such that $\overline{f^{\prime}}\left(\gamma_{k^{\prime \prime}}^{+}\right) \subset \gamma_{k}^{+}$.

If $\gamma_{k}^{+} \cap \partial(\operatorname{cl}(N \backslash L))=\emptyset$ then $\overline{f^{\prime}}\left(\gamma_{k}^{+}\right)=\gamma_{k^{\prime}}^{+}$. In fact, if this is not true, using the above lemma we can obtain a small enough connected set $\gamma$ with $\overline{f^{\prime}}\left(\gamma_{k}^{+}\right) \subset \gamma \subset \gamma_{k^{\prime}}^{+}$, $\gamma \neq \overline{f^{\prime}}\left(\gamma_{k}^{+}\right)$, and such that $\overline{f^{\prime}}-1(\gamma) \subset N_{L}$ is connected (homeomorphic to $\gamma$ ). Then $\gamma_{k}^{+} \subset$ ${\overline{f^{\prime}}}^{-1}(\gamma) \subset H_{p}^{+} \backslash\{p\}$ with $\gamma_{k}^{+} \neq{\overline{f^{\prime}}}^{-1}(\gamma)$ connected, and this is impossible. It is clear that $\gamma_{k^{\prime}}^{+} \cap \partial(\operatorname{cl}(N \backslash L))=\emptyset$.

As a consequence we obtain that if $\gamma_{k}^{+} \cap \partial(\operatorname{cl}(N \backslash L)) \neq \emptyset$, the component $\gamma_{k^{\prime \prime}}^{+}$intersects $\partial(\operatorname{cl}(N \backslash L))$.

Let us suppose that there exists a component $\gamma_{k}^{+}$of $H_{p}^{+} \backslash\{p\}$ with $\gamma_{k}^{+} \cap \partial(\operatorname{cl}(N \backslash L))=\emptyset$. Then, since $\operatorname{Inv}(\operatorname{cl}(N \backslash L), f)=\{p\}$, we have that the number of such components is infinite.

On the other hand, in order to see that the number of components which intersect $\partial(\operatorname{cl}(N \backslash L))$ is also infinite, we have only to observe that there exists a sequence of components of $H_{p}^{+} \backslash\{p\},\left\{\gamma_{n}\right\}_{n \in \mathbb{Z}}$, such that $\gamma_{0}=\gamma_{k}^{+}$and $\overline{f^{\prime}}\left(\gamma_{n}\right) \subset \gamma_{n+1}$. We have that $\gamma_{n} \cap \partial(\operatorname{cl}(N \backslash L))=\emptyset$ for every $n \geq 0$ and, since $\operatorname{Inv}(\operatorname{cl}(N \backslash L), f)=\{p\}$, there exists $n_{0} \in \mathbb{N}$ such that $\gamma_{-n_{0}} \cap \partial(\operatorname{cl}(N \backslash L)) \neq \emptyset$. Then, if the number of components which intersect $\partial(\operatorname{cl}(N \backslash L))$ is finite, the set of components $\left\{\gamma_{n}\right\}_{n \leq-n_{0}}$ is finite and we have automatically a contradiction.

Proposition 7. Let $f: U \subset \mathbb{R}^{2} \rightarrow f(U) \subset \mathbb{R}^{2}$ be a homeomorphism and let $p$ be a fixed point of $f$ such that $\{p\}$ is an isolated invariant set which is neither an attractor nor a repeller. Let us consider a strong filtration pair $(N, L)$ of period $r$ such that $\{p\}=\operatorname{Inv}(\operatorname{cl}(N \backslash L), f)$. Then there exist trivial shape continua $S_{1}, \ldots, S_{s}, U_{1}, \ldots, U_{s}$ in $\operatorname{cl}(N \backslash L)$, with $s=1-i_{\mathbb{R}^{2}}\left(f^{r}, p\right)$, such that:

(1) $\bigcup_{i=1}^{s} S_{i} \subset H_{p}^{+}$and $\bigcup_{i=1}^{s} U_{i} \subset H_{p}^{-}$;

(2) $S_{i} \cap S_{j}=U_{i} \cap U_{j}=\{p\}$ for all $i \neq j$ and $S_{i} \cap U_{j}=\{p\}$ for all $i, j \in\{1, \ldots, s\}$;

(3) $f^{r}\left(S_{i}\right) \subset S_{i}, f^{-r}\left(U_{i}\right) \subset U_{i}$;

(4) the sets $S_{i} \cap \partial(\operatorname{cl}(N \backslash L))$ and $U_{i} \cap \partial(\operatorname{cl}(N \backslash L))$ alternate in $\partial(\operatorname{cl}(N \backslash L))$.

Proof. Let $H_{p}^{-} \backslash\{p\}=\bigcup \gamma_{m}^{-}$where $\gamma_{m}^{-}$are the components of $H_{p}^{-} \backslash\{p\}$. As in Proposition 6 we have that $\operatorname{cl}\left(\gamma_{m}^{-}\right)=\gamma_{m}^{-} \cup\{p\}$.

Let us consider a component of $H_{p}^{-} \backslash\{p\}, \gamma_{m(i)}^{-} \subset \pi^{-1}\left(A\left(p_{i}\right)\right)$ such that $\gamma_{m(i)}^{-} \cap$ $\partial_{N}\left(L_{i}\right) \neq \emptyset$ (see the Main Theorem for a proof of the existence of $\gamma_{m(i)}^{-}$).

We define $U_{i}=\bigcup_{n \in \mathbb{N}} f^{-n r}\left(\operatorname{cl}\left(\gamma_{m(i)}^{-}\right)\right)$. Since $\bigcap_{n} f^{-n r}\left(H_{p}^{-}\right)=\{p\}$ it is not difficult to see that $U_{i} \subset H_{p}^{-}$is a trivial shape continuum.

We have $f^{-r}\left(U_{i}\right) \subset U_{i}, p \in U_{i}$ and

$$
U_{i} \cap \partial(\operatorname{cl}(N \backslash L))=\gamma_{m(i)}^{-} \cap \partial(\operatorname{cl}(N \backslash L)) \subset \partial_{N}\left(L_{i}\right) .
$$

If we repeat this construction for $i \in\{1, \ldots, s\}$ we obtain $U_{1}, \ldots, U_{s}$ with $U_{i} \cap U_{j}=$ $\{p\}$ for every $i \neq j$. 
Let us construct the sets $S_{1}, \ldots, S_{s} . H_{p}^{+} \backslash\{p\}=\bigcup \gamma_{m}^{+}$where $\gamma_{m}^{+}$are the components of $H_{p}^{+} \backslash\{p\}$. We have that $\operatorname{cl}\left(\gamma_{m}^{+}\right)=\gamma_{m}^{+} \cup\{p\}$. Let us consider the set $\theta=\left\{p_{1}, \ldots, p_{s}\right\}$ with $p_{i}$ adjacent to $p_{i+1}$.

We define $K_{i}=\bigcap_{n \in \mathbb{N}}\left(\overline{f^{\prime}}\right)^{n r}\left(\operatorname{cl}\left(A\left(p_{i}\right)\right)\right)$ for every $i \in\{1, \ldots, s\}$. Since $\left(\overline{f^{\prime}}\right)^{r}$ $\left(\operatorname{cl}\left(A\left(p_{i}\right)\right)\right) \subset \operatorname{cl}\left(A\left(p_{i}\right)\right)$, it is clear that $K_{i}$ is a continuum with $\left\{p, p_{i}\right\} \subset K_{i}=\left(\overline{f^{\prime}}\right)^{r}\left(K_{i}\right)$ $\subset \operatorname{cl}\left(A\left(p_{i}\right)\right)$.

If $\overline{p_{i-1} p_{i}}$ is the arc in $\partial(\operatorname{cl}(N \backslash L)) / \sim$ which makes adjacent $p_{i-1}$ and $p_{i}$, we have that there is a component $\gamma_{m(i)}^{+}$of $H_{p}^{+} \backslash\{p\}$ which intersects $K_{p_{i}}$ (the component of $\partial\left(A\left(p_{i}\right)\right)$ separating $p_{i}$ from $\theta \backslash p_{i}$, see the proof of the Main Theorem) and $\partial(\operatorname{cl}(N \backslash L)) \cap \gamma_{m(i)}^{+} \subset \pi^{-1}\left(\overline{p_{i-1} p_{i}}\right)$.

Let us consider the component $B_{i}$ of $N_{L} \backslash \bigcup_{i=1}^{s} K_{i}$ which contains $\gamma_{m(i)}^{+}$. Since $\left(\overline{f^{\prime}}\right)^{r}$ $\left(K_{i}\right)=K_{i}$ (with $f^{r}$ orientation-preserving) for every $i \in\{1, \ldots, s\}$, then it is not difficult to see that $\overline{\left(f^{\prime}\right)^{r}}\left(\left(H_{p}^{+} \backslash\{p\}\right) \cap B_{i}\right) \subset B_{i}$.

Using that $\bigcap_{n} f^{n r}\left(H_{p}^{+}\right)=\{p\}$ it is easy to check that

$$
S_{i}=\bigcup_{n \in \mathbb{N}} f^{n r}\left(\operatorname{cl}\left(\gamma_{m(i)}^{+}\right)\right) \subset H_{p}^{+} \cap \operatorname{cl}\left(B_{i}\right)
$$

are trivial shape continua for $i \in\{1, \ldots, s\}$.

We have $f^{r}\left(S_{i}\right) \subset S_{i}, p \in S_{i}$ and

$$
S_{i} \cap \partial(\operatorname{cl}(N \backslash L))=\gamma_{m(i)}^{+} \cap \partial(\operatorname{cl}(N \backslash L)) .
$$

If we make this construction for $i \in\{1, \ldots, s\}$, we obtain $S_{1}, \ldots, S_{s}$. Since $B_{i} \cap B_{j}=\varnothing$ for $i \neq j$ and $S_{i} \subset\{p\} \cup B_{i}$ we have that $S_{i} \cap S_{j}=\{p\}$ for every $i \neq j$.

It is obvious that the sets $S_{i} \cap \partial(\operatorname{cl}(N \backslash L))$ and $U_{i} \cap \partial(\operatorname{cl}(N \backslash L))$ alternate in $\partial(\operatorname{cl}(N \backslash L))$ and $U_{i} \cap S_{j}=\{p\}$ for every $i, j \in\{1, \ldots, s\}$.

Proposition 8. Let $f: U \subset \mathbb{R}^{2} \rightarrow f(U) \subset \mathbb{R}^{2}$ be a homeomorphism and let $p$ be a fixed point of $f$ such that $\{p\}$ is an isolated invariant set which is neither an attractor nor a repeller. Let us consider a strong filtration pair $(N, L)$ of period $r$ such that $\{p\}=\operatorname{Inv}(\operatorname{cl}(N \backslash L), f)$. If the number of components of $H_{p}^{+} \backslash\{p\}$ is finite, then there are exactly $1-i_{\mathbb{R}^{2}}\left(f^{r}, p\right)$ components.

Proof. In this situation we have that every component of $H_{p}^{+} \backslash\{p\}$ intersects $\partial(\operatorname{cl}(N \backslash L))$. For each component $\gamma_{m}^{+}$there exists $\gamma_{m^{\prime \prime}}^{+}$such that $\overline{f^{\prime}}\left(\gamma_{m^{\prime \prime}}^{+}\right) \subset \gamma_{m}^{+}$and $\overline{f^{\prime}}\left(\gamma_{m}^{+}\right) \subset \gamma_{m^{\prime}}^{+}$ for some component $\gamma_{m^{\prime}}^{+}$. Then $H_{p}^{+} \backslash\{p\}$ decomposes in a finite union of components on which $\overline{f^{\prime}}$ acts as a permutation. Let us consider $A\left(p_{i}\right)$ with $p_{i} \in\left\{p_{1}, \ldots, p_{s}\right\}$ where $p_{j}$ and $p_{j+1}$ are adjacent for every $j \in\{1, \ldots, s-1\}$. We have that there exist two components $\gamma_{m(i)}^{+}, \gamma_{m^{\prime}(i)}^{+}$of $H_{p}^{+} \backslash\{p\}$ such that $K_{p_{i}} \subset \operatorname{cl}\left(\gamma_{m(i)}^{+}\right) \cup \operatorname{cl}\left(\gamma_{m^{\prime}(i)}^{+}\right)$. Let us

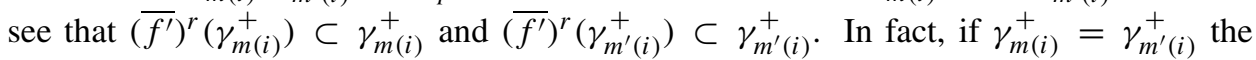
result follows automatically because, in this case, $H_{p}^{+} \backslash\{p\}=\gamma_{m(i)}^{+}$. If $\gamma_{m(i)}^{+} \neq \gamma_{m^{\prime}(i)}^{+}$, following the proof of Proposition 7 we have $\gamma_{m(i)}^{+} \subset B_{i}$ and $\gamma_{m^{\prime}(i)}^{+} \subset B_{i+1}$ with $B_{i} \neq B_{i+1}$. Since $\left(\overline{f^{\prime}}\right)^{r}\left(K_{p_{i}}\right) \subset K_{p_{i}}$ and $\left(\overline{f^{\prime}}\right)^{r}\left(\left(H_{p}^{+} \backslash\{p\}\right) \cap B_{i}\right) \subset B_{i}$ for $i \in\{1, \ldots, s\}$ we have that the result is proved. Then there is no $\gamma_{m^{\prime \prime}}^{+} \notin\left\{\gamma_{m(i)}^{+}, \gamma_{m^{\prime}(i)}^{+}\right\}$such that $\left(\overline{f^{\prime}}\right)^{r}\left(\gamma_{m^{\prime \prime}}^{+}\right) \subset \gamma_{m(i)}^{+} \cup \gamma_{m^{\prime}(i)}^{+}$. 
Let $\gamma_{m}^{+} \notin\left\{\gamma_{m(1)}^{+}, \gamma_{m^{\prime}(1)}^{+}, \ldots, \gamma_{m(s)}^{+}, \gamma_{m^{\prime}(s)}^{+}\right\}$. Since each $x \in A$ is such that there is $n_{0} \in \mathbb{N}$ with $\left(\overline{f^{\prime}}\right)^{n_{0}}(x) \in A\left(p_{i}\right)$ for some $i \in\{1, \ldots, s\}$, then it is easy to prove that $\left(\overline{f^{\prime}}\right)^{n r}\left(\gamma_{m}^{+}\right) \subset \gamma_{m(i)}^{+} \cup \gamma_{m^{\prime}(i)}^{+}$for $n \in \mathbb{N}$ big enough and for some $i \in\{1, \ldots, s\}$, and this contradicts the last paragraph. Then we have that there is not such a $\gamma_{m}^{+}$.

Since $\left\{p_{1}, \ldots, p_{s}\right\}$ are adjacent in $\partial(\operatorname{cl}(N \backslash L)) / \sim$ we have, by the same arguments, that $\gamma_{m^{\prime}(i)}^{+}=\gamma_{m(i+1)}^{+}$, and the result is proved.

As a consequence of Theorem 3 and the Main Theorem we have the following.

COROllary 4. Let $f: U \subset \mathbb{R}^{2} \rightarrow f(U) \subset \mathbb{R}^{2}$ be a homeomorphism. Let $p \in U$ be a fixed point that is the unique periodic point of $f$. Suppose that there is a topological disk $D$ such that $p \in \operatorname{Inv}(D, f) \subset \operatorname{int}(D)$ and that there is not a disk $B \subset D$ containing $p$ such that $B \subset \operatorname{int}(f(B))$. Then, there are $r \in \mathbb{N}$ and a disk $N$ such that the components $H_{p}^{+}$, of $\operatorname{Inv}^{+}(N, f)$, and $H_{p}^{-}$, of $\operatorname{Inv}^{-}(N, f)$, containing $p$, are trivial shape continua such that $\lim _{k \rightarrow \infty} f^{k}(x)=p$ for every $x \in H_{p}^{+}, \lim _{k \rightarrow \infty} f^{-k}(x)=p$ for every $x \in H_{p}^{-}$and $H_{p}^{+} \cap \partial(N)$ and $H_{p}^{-} \cap \partial(N)$ have at least $1-i_{\mathbb{R}^{2}}\left(f^{r}, p\right)$ components.

Combining Theorem 3, the Main Theorem and Proposition 7 we obtain the following.

COROllary 5. Let $f: U \subset \mathbb{R}^{2} \rightarrow f(U) \subset \mathbb{R}^{2}$ be a local homeomorphism. Let $p \in U$ be a fixed point of $f$ such that $\{p\}$ is an isolated invariant set which is neither an attractor nor a repeller. Then, there are $r \geq 1$ and a neighborhood system of $p,\left\{B_{m}\right\}_{m \in \mathbb{N}}$, such that if $H_{p}^{+m}$ and $H_{p}^{-m}$ are the components of $\operatorname{Inv}^{+}\left(B_{m}, f\right)$ and $\operatorname{Inv}^{-}\left(B_{m}, f\right)$ respectively containing $p, H_{p}^{+m}$ and $H_{p}^{-m}$ are trivial shape continua such that $\lim _{k \rightarrow \infty} f^{k}(x)=p$ for every $x \in H_{p}^{+m}$ and $\lim _{k \rightarrow \infty} f^{-k}(x)=p$ for every $x \in H_{p}^{-m}$, and $H_{p}^{+m} \cap \partial\left(B_{m}\right)$ and $H_{p}^{-m} \cap \partial\left(B_{m}\right)$ have at least $1-i_{\mathbb{R}^{2}}\left(f^{r}, p\right)$ components, for every $m \in \mathbb{N}$. Moreover, there exist trivial shape continua $S_{1}^{m}, \ldots, S_{s}^{m}, U_{1}^{m}, \ldots, U_{s}^{m}$ in $B_{m}$, with $s=1-i_{\mathbb{R}^{2}}\left(f^{r}, p\right)$, such that:

(1) $\bigcup_{i=1}^{s} S_{i}^{m} \subset H_{p}^{+m}$ and $\bigcup_{i=1}^{s} U_{i}^{m} \subset H_{p}^{-m}$;

(2) $S_{i}^{m} \cap S_{j}^{m}=U_{i}^{m} \cap U_{j}^{m}=\{p\}$ for all $i \neq j$ and $S_{i}^{m} \cap U_{j}^{m}=\{p\}$ for all $i, j \in\{1, \ldots, s\}$;

(3) $f^{r}\left(S_{i}^{m}\right) \subset S_{i}^{m}, f^{-r}\left(U_{i}^{m}\right) \subset U_{i}^{m}$;

(4) the sets $S_{i}^{m} \cap \partial\left(B_{m}\right)$ and $U_{i}^{m} \cap \partial\left(B_{m}\right)$ alternate in $\partial\left(B_{m}\right)$.

Remark 3. Le Calvez, in [9], proved that if $f: U \subset \mathbb{R}^{2} \rightarrow f(U) \subset \mathbb{R}^{2}$ is an orientationpreserving homeomorphism, $p \in U$ is the unique fixed point of $f$ and $D \subset U$ is a disk such that $p \in \operatorname{int}(D)$, then if $\operatorname{int}\left(\operatorname{Inv}^{+}(D, f)\right)=\operatorname{int}\left(\operatorname{Inv}^{-}(D, f)\right)=\emptyset$ it follows that there are a disk $D^{\prime} \subset D$ and a homeomorphism $f^{*}: U \subset \mathbb{R}^{2} \rightarrow \mathbb{R}^{2}$ such that $\operatorname{Fix}\left(f^{*}\right) \cap U=$ $\{p\}, p \in \operatorname{Inv}\left(D^{\prime}, f^{*}\right) \subset \operatorname{int}\left(D^{\prime}\right), f^{*}$ is uniformly near to $f$ and $f^{*}=f$ in a neighborhood of $p$. Therefore, as a consequence, using Theorem 3 , if $i_{\mathbb{R}^{2}}(f, p)=-n$ and there is a disk $D \subset U$ with $p \in \operatorname{int}(D)$ such that $\operatorname{int}\left(\operatorname{Inv}^{+}(D, f)\right)=\operatorname{int}\left(\operatorname{Inv}^{-}(D, f)\right)=\emptyset$, we can find a sequence of similar homeomorphisms $\left\{f_{m}\right\} \rightarrow f$ such that all of them admit strong filtration pairs of period 1 . We can apply the Main Theorem to each $f_{m}$ to get sequences of compacta $\left\{K_{m}^{+}\right\} \rightarrow K^{+}$and $\left\{K_{m}^{-}\right\} \rightarrow K^{-}$, with the upper semicontinuous topology. In particular, if $f$ is area-preserving then $\operatorname{int}\left(\operatorname{Inv}^{+}(D, f)\right)=\operatorname{int}\left(\operatorname{Inv}^{-}(D, f)\right)=\emptyset$ and one can apply the above argument (see Theorem 1 and [1]). 
Acknowledgements. The first author wishes to thank the Mathematical Institute of the UNAM at Cuernavaca for its hospitality and support. We also are indebted to Professor M. A. Morón for his comments and to Professor R. Ortega for sharing his knowledge with us and proposing to us some of the problems we studied here. Finally, we also wish to acknowledge the referee's suggestions which have been useful in improving the first version of the paper. The first author has been supported by MCyT, BMF 2000-0804-C03-01.

\section{A. Appendix}

Definition A1. A compact set $N$ is called an isolating block if

$$
f(N) \cap N \cap f^{-1}(N) \subset \operatorname{int}(N) .
$$

Definition A2. (See [15]) Let $S$ be an isolated invariant set and suppose $L \subset N$ is a compact pair contained in the interior of the domain of $f$. The pair $(N, L)$ is called a filtration pair for $S$ provided $N$ and $L$ are each the closure of their interiors and

(1) $\operatorname{cl}(N \backslash L)$ is an isolating neighborhood of $S$;

(2) $L$ is a neighborhood of $N^{-}$in $N$; and

(3) $f(L) \cap \operatorname{cl}(N \backslash L)=\emptyset$.

THEOREM A1. Let $f: U \subset \mathbb{R}^{2} \rightarrow f(U) \subset \mathbb{R}^{2}$ be a homeomorphism and let us suppose that there is a disk $D \subset U$ such that $\operatorname{Fix}(f) \cap D=\{p\}$ and $\operatorname{Inv}(D, f) \subset \operatorname{int}(D)$. Then, there exists an isolating block $N$, such that $N$ is homeomorphic to a disk and $\operatorname{Inv}(N, f) \subset \operatorname{Inv}(D, f)$ and $p \in \operatorname{Inv}(N, f) \subset \operatorname{int}(N)$.

Proof. We give a proof shorter than that of [21], based on the following argument that seems to be due to Yoccoz.

There exist an integer $k_{0} \geq 1$ such that $\bigcap_{-k_{0} \leq k \leq k_{0}} f^{-k}(D) \subset \operatorname{int}(D)$. Let us consider a family of disks $\left\{D_{k}\right\}_{0 \leq k \leq k_{0}}$ such that

$$
D_{0}=D, D_{k} \subset \operatorname{int}\left(D_{k+1}\right) \quad \text { and } \bigcap_{-k_{0} \leq k \leq k_{0}} f^{-k}\left(D_{k_{0}}\right) \subset \operatorname{int}\left(D_{0}\right)
$$

The connected component $U$ of $\bigcap_{-k_{0} \leq k \leq k_{0}} f^{-k}\left(D_{|k|}\right)$ that contains $p$ is a Jordan domain, by a well-known theorem of Kerékjártó (see [18]), which means that its adherence is a disk $N$. The set $X=\bigcap_{-k_{0} \leq k \leq k_{0}} f^{-k}\left(D_{|k|}\right)$ is an isolating block. Indeed,

$$
\begin{aligned}
f^{-1}(X) \cap X \cap f(X) & =\bigcap_{-k_{0} \leq k \leq k_{0}} f^{-k-1}\left(D_{|k|}\right) \bigcap_{-k_{0} \leq k \leq k_{0}} f^{-k}\left(D_{|k|}\right) \bigcap_{-k_{0} \leq k \leq k_{0}} f^{-k+1}\left(D_{|k|}\right) \\
& \subset \bigcap_{0 \leq k \leq k_{0}-1} f^{-k-1}\left(D_{k}\right) \bigcap_{-k_{0} \leq k \leq k_{0}} f^{-k}\left(D_{k_{0}}\right) \bigcap_{-k_{0}+1 \leq k \leq 0} f^{-k+1}\left(D_{-k}\right) \\
& \subset \bigcap_{1 \leq k \leq k_{0}} f^{-k}\left(\operatorname{int}\left(D_{k}\right)\right) \bigcap \operatorname{int}\left(D_{0}\right) \bigcap_{-k_{0} \leq k \leq 1} f^{-k}\left(\operatorname{int}\left(D_{-k}\right)\right) \subset \operatorname{int}(X) .
\end{aligned}
$$

The disk $N$ is itself an isolating block because

$$
f^{-1}(N) \cap N \cap f(N) \subset \operatorname{int}(X) \cap N \subset \operatorname{int}(N) .
$$


THEOREM A2. Let $f: U \subset \mathbb{R}^{2} \rightarrow \mathbb{R}^{2}$ be a homeomorphism. Let us assume that there is a disk $D \subset U$ such that $\operatorname{Fix}(f) \cap D=\{p\}, \operatorname{Inv}(D, f) \subset \operatorname{int}(D)$ and that there is not a disk $B \subset D$ containing $p$ such that $B \subset \operatorname{int}(f(B))$. Then, there exists a filtration pair $(N, L)$ such that $p \in \operatorname{Inv}(\operatorname{cl}(N \backslash L), f)$ and $N$ and the components of $L$ are topological disks.

Proof. Let $N$ be the isolating block constructed in the above theorem. Using Theorem 3.7 in [15], there is $L=L_{1} \cup \cdots \cup L_{n}$, such that $(N, L)$ is a filtration pair and $L$ is a twodimensional manifold. Then, $L$ is a finite disjoint union of $n$ disks, each of them having a finite amount of holes.

Given the disk $G$, a hole of $L_{i}$, first, we will check that $p \notin G$.

Indeed, otherwise we can take the smallest hole, $G_{0}$, of all those containing $p$. Since $f(L) \cap \operatorname{cl}(N \backslash L)=\emptyset$, it follows that $f\left(\partial\left(G_{0}\right)\right) \cap \operatorname{cl}(N \backslash L)=\emptyset$. Since there is not a disk $B$ containing $p$ such that $B \subset \operatorname{int}(f(B))$ one gets immediately a contradiction.

Now, take $(N, D(L))$, where $D(L)$ is the family of disks obtained by filling the holes of $L$. In order to check that $(N, D(L))$ is a filtration pair, only a proof of the fact that $f(D(L)) \cap \operatorname{cl}(N \backslash D(L))=\emptyset$ is needed.

Let $\left\{D\left(L_{1}\right), \ldots, D\left(L_{n}\right)\right\}$ be the disks obtained by filling the holes of $L_{i}$. Then $D(L)=$ $\bigcup_{i=1}^{n} D\left(L_{i}\right)$.

Since $\operatorname{cl}(N \backslash D(L)) \subset \operatorname{cl}(N \backslash L)$ we have that $f(L) \cap \operatorname{cl}(N \backslash D(L))=\emptyset$.

Let us write $\operatorname{cl}\left(D\left(L_{i}\right) \backslash L_{i}\right)=D_{1}^{i} \cup \cdots \cup D_{p(i)}^{i}$ which is a finite union of disks.

Then,

$$
f(D(L)) \cap \operatorname{cl}(N \backslash D(L))=\left(f\left(\bigcup_{j=1}^{p(1)} D_{j}^{1}\right) \cup \cdots \cup f\left(\bigcup_{j=1}^{p(n)} D_{j}^{n}\right)\right) \cap \operatorname{cl}(N \backslash D(L)) .
$$

Since $\partial\left(D_{j}^{r}\right) \subset \partial_{N}(L)$, from the properties of filtration pair it follows that

$$
f\left(\partial\left(D_{j}^{r}\right)\right) \subset \operatorname{int}(L) \subset \operatorname{int}(D(L)) .
$$

Therefore $f\left(D_{j}^{r}\right) \subset \operatorname{int}(D(L))$. Consequently, $f\left(D_{j}^{r}\right) \cap \operatorname{cl}(N \backslash D(L))=\emptyset$ and $f(D(L)) \cap$ $\operatorname{cl}(N \backslash D(L))=\emptyset$.

Proof of Theorem 3. Take $(N, L)$, a filtration pair for $\operatorname{Inv}(N, f)$, as in the above theorem. $N$ is a disk which is an isolating block, and $L=L_{1} \cup \cdots \cup L_{n}$ is a disjoint union of disks. Without lost of generality we can assume that $L_{j} \cap N^{-} \neq \emptyset$ for every $j \in\{1, \ldots, n\}$.

Divide $\left\{L_{1}, \ldots, L_{n}\right\}$ into two classes:

(1) the $L_{i}$ such that $N \backslash L_{i}$ is not connected; we will say that these $L_{i}$ are transversal with respect to $N$;

(2) the $L_{i}$ such that $N \backslash L_{i}$ is connected.

Denote by $\left\{L_{1}, \ldots, L_{p}\right\}$ the family of the transversal components of $L$ that can be connected with $p$ using a path in $N$ without intersection with any other transversal component of $L$ and let $\left\{L_{p+1}, \ldots, L_{m}\right\}$ be the family of non-transversal components of $L$ that can be connected with $p$ using a path in $N$ without intersection with any other transversal component of $L$. 
For each $i \in\{1, \ldots, p\}$ define

$$
\overline{L_{i}}=N \backslash \text { c.c. }\left(N \backslash L_{i}, p\right)
$$

where c.c. $\left(N \backslash L_{i}, p\right)$ is the component of $N \backslash L_{i}$ containing $p$.

Consider the pair $(N, \bar{L})$ with

$$
\bar{L}=\left(\bigcup_{i=1}^{p} \overline{L_{i}}\right) \cup\left(\bigcup_{i=p+1}^{m} L_{i}\right) .
$$

Each component of $\bar{L}$ is a non-transversal disk and $(N, \bar{L})$ is a strong filtration pair such that $p \in \operatorname{Inv}(\operatorname{cl}(N \backslash \bar{L}), f) \subset \operatorname{Inv}(D, f)$.

\section{REFERENCES}

[1] S. Baldwin and E. E. Slaminka. A stable/unstable 'manifold' theorem for area preserving homeomorphisms of two dimensions. Proc. Amer. Math. Soc. 109(3) (1990), 823-828.

[2] M. Bonino. Lefschetz index for orientation reversing planar homeomorphisms. Proc. Amer. Math. Soc. 130(7) (2002), 2173-2177.

[3] K. Borsuk. Theory of Shape (Monografie Matematyczne, 59). PWN, Warsaw, 1975.

[4] K. Borsuk. Theory of Retracts (Monografie Matematyczne, 44). PWN, Warsaw, 1967.

[5] L. E. Brouwer. Beweis des ebenen translationssatzes. Math. Ann. 72 (1912), 37-54.

[6] M. Brown. On the fixed point index of iterates of planar homeomorphisms. Proc. Amer. Math. Soc. 108 (1990), 1109-1114.

[7] M. Brown. A new proof of Brouwer's lemma on translation arcs. Houston J. Math. 10 (1984), 35-41.

[8] R. F. Brown. The Lefschetz Fixed Point Theorem. Scott Foreman, Glenview, IL and London, 1971.

[9] P. Le Calvez. Une propriété dynamique des homéomorphismes du plan au voisinage d'un point fixe d'indice >1. Topology 38(1) (1999), 23-35.

[10] P. Le Calvez and J. C. Yoccoz. Un théoréme d'indice pour les homéomorphismes du plan au voisinage d'un point fixe. Ann. Math. 146 (1997), 241-293.

[11] C. O. Christenson and W. L. Voxman. Aspects of Topology. BCS Associates, Moscow, ID, 1998.

[12] E. N. Dancer and R. Ortega. The index or Lyapunov stable fixed points. J. Dynam. Differential Equations 6 (1994), 631-637.

[13] A. Dold. Fixed point index and fixed point theorem for Euclidean neighborhood retracts. Topology 4 (1965), 1-8.

[14] J. Franks. The Conley index and non-existence of minimal homeomorphisms. Illinois J. Math. 43(3) (1999), 457-464.

[15] J. Franks and D. Richeson. Shift equivalence and the Conley index. Trans. Amer. Math. Soc. 352(7) (2000), 3305-3322.

[16] M. Handel. There are no minimal homeomorphisms of the multipunctured plane. Ergod. Th. \& Dynam. Sys. 12 (1992), 75-83.

[17] M. W. Hirsch. Fixed-point indices, homoclinic contacts, and dynamics of injective planar maps. Michigan Math. J. 47 (2000), 101-108.

[18] B. Kerékjártó. Voresungen über Topologie (I). Springer, Berlin, 1923.

[19] R. D. Nussbaum. The Fixed Point Index and some Applications. Séminaire de Mathématiques supérieures, Les Presses de L'Université de Montréal, 1985.

[20] S. Pelikan and E. E. Slaminka. A bound for the fixed point index of area-preserving homeomorphisms of two-manifolds. Ergod. Th. \& Dynam. Sys. 7 (1987), 463-479.

[21] F. R. Ruiz del Portal and J. M. Salazar. Fixed point index of iterations of local homeomorphisms of the plane: a Conley-index approach. Topology 41 (2002), 1199-1212.

[22] M. Shub and D. Sullivan. A remark on the Lefschetz fixed point formula for differentiable maps. Topology 13 (1974), 189-191.

[23] C. P. Simon. A bound for the fixed point index of an area-preserving map with applications to mechanics. Invent. Math. 26 (1974), 187-200. 